\title{
Conductivity Profiling of Graphite Moderator Bricks from Multi-Frequency Eddy Current Measurements
}

DOI:

10.1109/JSEN.2020.2965201

\section{Document Version}

Accepted author manuscript

Link to publication record in Manchester Research Explorer

\section{Citation for published version (APA):}

Tesfalem, H., Peyton, A., Fletcher, A., Brown, M., \& Chapman, B. (2020). Conductivity Profiling of Graphite Moderator Bricks from Multi-Frequency Eddy Current Measurements. IEEE Sensors Journal.

https://doi.org/10.1109/JSEN.2020.2965201

\section{Published in:}

IEEE Sensors Journal

\section{Citing this paper}

Please note that where the full-text provided on Manchester Research Explorer is the Author Accepted Manuscript or Proof version this may differ from the final Published version. If citing, it is advised that you check and use the publisher's definitive version.

\section{General rights}

Copyright and moral rights for the publications made accessible in the Research Explorer are retained by the authors and/or other copyright owners and it is a condition of accessing publications that users recognise and abide by the legal requirements associated with these rights.

\section{Takedown policy}

If you believe that this document breaches copyright please refer to the University of Manchester's Takedown Procedures [http://man.ac.uk/04Y6Bo] or contact uml.scholarlycommunications@manchester.ac.uk providing relevant details, so we can investigate your claim.

\section{OPEN ACCESS}




\title{
Conductivity Profiling of Graphite Moderator Bricks from Multi-Frequency Eddy Current Measurements
}

\author{
Henok Tesfalem, Anthony J Peyton, Adam D Fletcher, Matthew Brown and Bob Chapman
}

\begin{abstract}
This paper presents a novel system for mapping the through-wall electrical conductivity profiles of graphite moderator bricks that make up the core of an Advanced GasCooled Reactor. Multi-frequency eddy current measurements were used to reconstruct the conductivity profiles as a function of depth from the bores of different graphite bricks. The study was carried out using a modified Levenberg Marquardt algorithm, along with a finite element based forward model. First, the algorithm was tested using a laboratory graphite sample with known electrical conductivity profile. Secondly, real datasets from one of the operating Advanced Gas-Cooled Reactors was collected and then used for reconstruction. The results in this study were compared with measurements from trepanned graphite samples taken from the reactor cores, and showed reasonable agreement in multiple cases, suggesting that this method could be a viable tool for non-destructively assessing the condition of the graphite core in Advanced Gas-Cooled Reactors.
\end{abstract}

Index Terms - Complex Structure, Eddy currents, Graphite, Conductivity, Multi-frequency, Non-Destructive Testing

\section{INTRODUCTION}

A dvanced Gas-cooled Reactors (AGRs) operate in the United Kingdom, contributing approximately $19 \%$ of the total annual electricity supplied to the country. AGRs use carbon dioxide as a coolant, and graphite brick cores to moderate the fast-moving neutrons, facilitate thermal energy transfer to the coolant gas and maintain the lattice spacing between the fuel assemblies and control rods [1]. The bricks which are in direct proximity to the fuel elements are subject to the highest radiation flux. As a result, they suffer most from irradiation and radiolytic oxidation [2]-[3].

Radiolytic oxidation of the fuel channel bricks is mainly caused by a chemical decomposition of the carbon dioxide coolant into carbon monoxide and oxidizing species when exposed to gamma radiation, which then attacks the graphite structure to increase the size of the pores, and hence leads to a reduction in graphite density or "weight loss" [2]-[3].

Anthony J Peyton, Henok Tesfalem and Adam D Fletcher are with School of Electrical and Electronic Engineering, University of Manchester, M13 9PL, UK. Matthew Brown is with EDF-Energy, Barnett Way, Barnwood, Gloucester, GL4 3RS, UK. Bob Chapman is an independent NDT Consultant, Gloucester, GL11 4EW, UK.

The work presented in this paper is funded by the UK Engineering and Physical Science Research Council and EDF Energy under a grant number EPSRC IAA 212.
Consequently, the oxidation could affect the core structural integrity margins and potentially reduce the operating lifetime of the reactors. The fuel channel bricks in the reactor core are not able to be replaced; therefore, the normal operation and safety functions of the AGRs depend significantly upon the condition of these bricks. For this reason, the assessment of AGR graphite properties is important to support the safety case for continued operation of the reactors.

Graphite is an electrically conductive material by nature. Therefore, eddy current (EC) based Non-Destructive Testing (NDT) could be used to characterise its internal properties, particularly as the graphite density reduction can be closely correlated with electrical conductivity [4]. Knowledge of graphite density gives an insight into its strength and residual moderating ability.

EC-based NDT is very well established, and its use within the civil nuclear industries is increasing significantly in recent years [5]. One of the driving factors for the use of EC-NDT in civil nuclear industries is its simplicity and robustness when deployed in radioactive environments, such as the cores of the AGR. However, the amount of information that can be obtained from an EC system could be limited if operated at a single frequency [6]. To overcome this limitation, a multifrequency (MF) excitation could be applied to excite the EC sensor. In this way full spectral information about the material of interest can be obtained [7]-[12]. In this context with an application of MF excitation technique, it is possible to probe the graphite fuel channel brick to different depths and collect enough data to characterise its electrical properties through its wall thickness.

However, one of the major challenges of material characterisation of the EC measurement, particularly depth profiling is that the measured data depends upon many factors including the material structure, sensor configuration, magnetic and electrical properties. Assuming an optimised sensor configuration, non-magnetic material with unknown electrical properties (which is the case for the problem considered in this paper), complex material structures still pose difficulties for depth profiling. This is because the field distribution within complex structures is less definite and requires robust inversion algorithms. Studies concerning inverse EC problem theory and applications have been published by many authors in the past, including [9]-[16]. Although these works lay the foundations for the inverse EC problems concerned with continuous, step and arbitrary material property variations along with the corresponding 
regularization techniques, most of them tend to focus on the cases where the inverse EC problem involve flat and relatively simple geometric structures. There exists other non-model based conductivity measurement approaches such as fourpoint potential drop [26], however this approach is only limited to bulk conductivity measurements and could not be applied for conductivity profiling as a function of depth. In recent years' machine learning approach has been used for different NDT applications such as defect detection and characterization [27], [28]. Machine learning approach has a potential for determining the material conductivity as a function of depth, but requires significant amount of training data.

For problems concerning nuclear graphite conductivity profiling, a study was carried out by Dekdouk et al. [16]. Although this study was made on flat graphite sections, it provided confidence for further development and refinement of this technique. Fletcher [17] extended the graphite conductivity profiling study to more realistic graphite bricks, demonstrating the practical feasibility of the method. A recent study by Tesfalem et.al [7], [18] demonstrated the ability of a MF EC system in achieving greater depth sensitivity through the entire radial thickness of a cylindrical graphite brick. The study in [18] also attempted to improve the conductivity profiling algorithm through the implementation of a priori knowledge based constraining technique using the measurements collected from a cylindrical laboratory sample.

In this paper, we extend our approach to the in-core fuel channel graphite brick reconstruction problem using a modified Regularized Levenberg Marquardt (RLM) algorithm, and present reconstructed conductivity profiles of AGR graphite bricks based on the measurements taken from one of the reactor cores in the UK's fleet of AGRs. The reconstruction process in this paper initially started with a simplified case using a simulated dataset containing different artificially added error levels, and the experimental data measured on a laboratory setup from a test sample with a known electrical conductivity profile. This was then followed by a reconstruction of real AGR fuel-channel bricks using the measurements collected during a maintenance outage from one of the operating AGRs. This approach may be of interest to the wider EC NDT and sensor community as it demonstrates that unknown properties of a hard-to-reach conducting material could be estimated from the EC measurements using an appropriate inversion method, which is particularly relevant for conductivity profiling of components with complex structures.

\section{BACKGROUND}

\section{A. Overview of the NDT problem}

EC based NDT works on the principle of electromagnetic induction. A coil carrying an alternating current generates a time-harmonic primary field around the coil that induces closed-loop EC into a conducting material placed within the field. The induced EC in turn generate a secondary field, which can be detected by a pick-up coil. Small changes in the electrical properties of the material of interest would alter the EC flow, and hence the detected signal. Therefore, this information could be used to estimate the electrical properties of the material using appropriate signal and data processing techniques.

The NDT of the graphite core in the AGRs is carried out from the brick bores using a specialized in-core inspection tool [5]. This tool is deployed from the top of the pile cap, and is fitted with gradiometer sensors equally separated circumferentially round the tool. The EC sensors in the tool are arranged to inspect the graphite brick over its radial cross section as well as to measure the density variation around the brick bore axially and circumferentially [5].

The tool has a rotating head enclosed by a cylindrical stainless steel casing, and uses non-conducting mechanical scissor-type arms to deploy the EC sensors towards the fuel channel bore. The inspections are made during outages of the

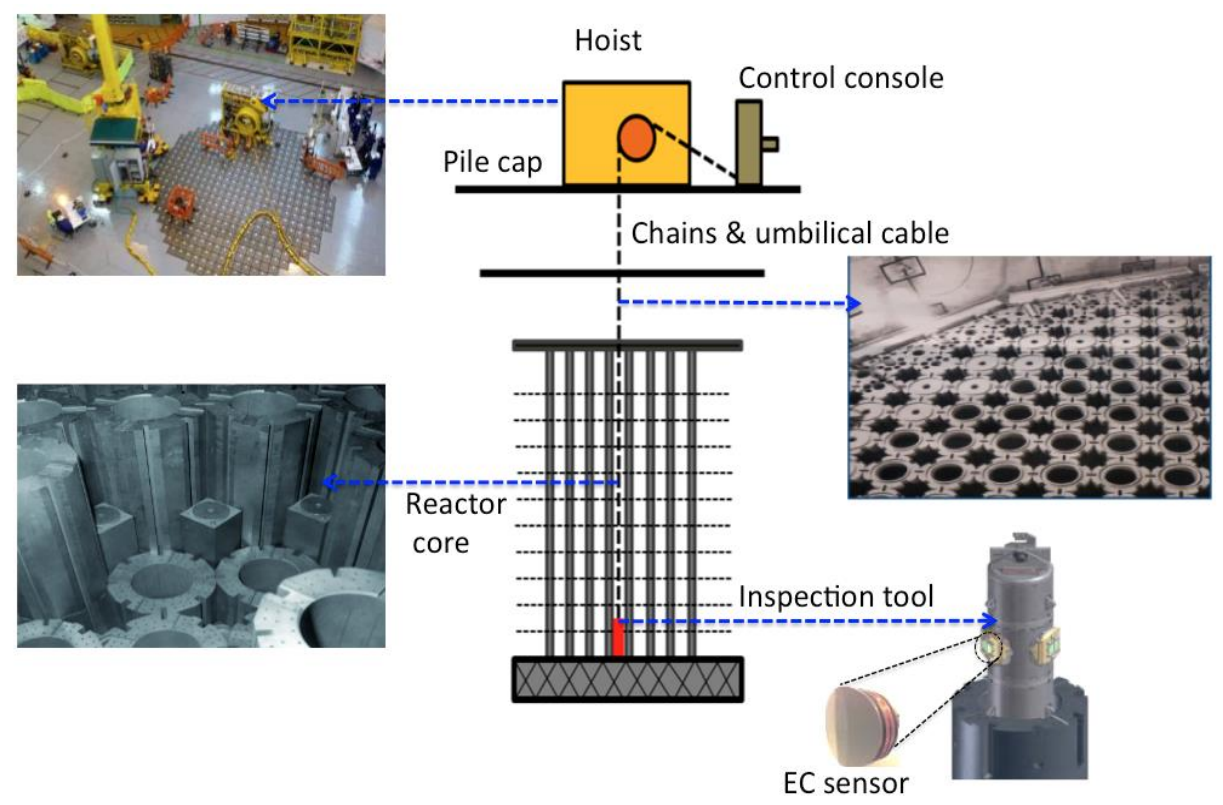

Fig. 1. Eddy current based AGR core inspection system along with illustration of the main core components and inspection procedures, reproduced from [5]. 
reactors while the fuel elements are removed. During the inspection the tool is lowered by a hoist into the bore of the fuel channel bricks using chains and an umbilical cable (see Fig. 1). The measurement speed, location and other inspection functions of the tool are set from the control console on the pile cap.

The major challenge of the NDT of graphite bricks in the AGR core is determining the radial conductivity variation as a function of distance from the bore. At present, the existing NDT systems can only monitor conductivity variations around the bore region. Depth information cannot be obtained nondestructively and is currently determined through the extraction of $\varnothing 19 \mathrm{~mm}$ by $75 \mathrm{~mm}$ long cylindrical samples trepanned from the core, which is approximately $80 \%$ of the brick radial thickness. The sample extraction method is essentially a destructive method, and it usually takes several months before the sample properties are determined from laboratory analysis. Furthermore, the conditions of the remaining $20 \%$ brick thickness cannot be assessed using the trepanning technique as the sample lengths are limited to 75 mm. Therefore, a new NDT method with a fast, efficient, depth-profiling capability is desirable. This in turn could be used for supporting the safety case for continued safe operation of the reactors.

\section{B. Conductivity profiling}

\section{1) Forward modeling}

The forward model plays a crucial part in obtaining an accurate solution for a given reconstruction problem. This is because the reconstruction problem seeks to minimize the discrepancies between the physically measured data and those predicted from the finite element (FE) forward model. Therefore, the forward model is often implemented such that it best represents the physical and electromagnetic properties of the material of interest and the measurement system. In some cases, additional model calibration procedures may be necessary using the measurement from a sample with known electrical properties.

The forward model for the problem considered in this paper is implemented using the commercial FE software, COMSOL Multiphysics. The Magnetic Field solver within the AC/DC module, along with an iterative approach known as the Flexible Generalized Minimum Residual (FGMRES) was used throughout the modeling process [19]. To increase the convergence rate of this solver a preconditioning Geometric Multigrid (GMG) was applied into the iterative solver, which aims to reduce the condition number of the stiffness matrix [19]. The boundary condition applied for this problem is the magnetic insulation boundary condition, which fixes the field variables being solved for (in this case the $\boldsymbol{A}$-field) to be zero on the selected boundaries. This boundary condition also ensures that the magnetic field is tangential to the boundary, and defines the symmetry condition, which has a mirror effect of the magnetic field.

This work employed the edge element formulation presented in [20]. The coils in the forward model were created as current-carrying filaments using the mean values of the practical coil dimensions, and the probe mutual inductance was calculated by computing the line integral of the $\boldsymbol{A}$-field around filaments representing the receiver coils (1).

$M=\frac{1}{I}\left(\oint \boldsymbol{A}_{P C} \cdot d \boldsymbol{l}-\oint \boldsymbol{A}_{B C} \cdot d \boldsymbol{l}\right)$

where $M$ is the mutual inductance of the gradiometer sensor, $I$ is the unit current applied into the exciter coil, $\mathbf{A}_{P C}$ and $\mathbf{A}_{B C}$ are the vector potential in the gradiometer Pick-up (PC) and Backing-off (BC) coils respectively. The graphite brick domain was discretised into seven and ten layers for the reactor brick and laboratory brick respectively. These is mainly to assign a conductivity value for each layer independently, that was subsequently updated through the iterative reconstruction process. To ease the computational demand, the entire problem domain was reduced to onequarter symmetry as shown in Fig. 2.

\section{2) Sensitivity calculation}

The sensitivity of the induced voltage in the sensor to variation in material properties is a crucial part of EC based NDT and also for solving inverse EC problems. Several authors in the electromagnetic NDT field have proposed different techniques for mapping the sensitivity of the test object both numerically and experimentally. These include the work by Yin et al. [9], which used the perturbation technique to map the change in coil inductance due to a small change in the conductivity of a test object. A more computationally efficient sensitivity formulation that can be applied to general electromagnetic problems was presented by Yin and Peyton [21] and Dyck et al. [22], and this formulation is adopted for the reconstruction problem considered in this paper. The sensitivity formulation in this paper is applied to map the change in graphite electrical conductivity $\delta \sigma$ to a change in boundary measurements $\delta M$ such that $s_{\sigma}=\frac{\delta M}{\delta \sigma}$.

Following the work by Dyck et.al. [22], the general form of the sensitivity to a change in the electrical conductivity of a graphite brick is given by (2).

$\boldsymbol{S}_{\sigma}=\int_{L_{i}}\left(\boldsymbol{E}_{T x} \cdot \boldsymbol{E}_{R x}\right) d v_{L_{i}}$

where $\boldsymbol{S}_{\sigma}$ is the sensitivity also known as the Jacobian matrix $\left(\boldsymbol{J}_{\sigma}\right), \quad \boldsymbol{E}_{T x}$ and $\boldsymbol{E}_{R x}$ are the electric field intensities resulting from excitation of the exciter coil and the receiver coils (in this case with a current that has opposite polarities), $v_{L_{i}}$ is the volume of the discretised graphite brick domain at $i^{\text {th }}$ layer (see Fig. 2) over which the dot product of the electric fields are integrated. It should be noted that (2) shows the general case of the sensitivity formulation for a single layer at a single frequency, and for the complete MF sensitivity matrix calculation the electric field intensities are evaluated for each frequency over each graphite layer.

\section{3) Inversion}

The graphite density loss (weight loss) caused by radiolytic oxidation is non-uniform through the brick cross-section. It is generally believed that the radiolytic oxidation rate is higher near the bore region and gradually decreases towards the outer brick periphery. This a priori knowledge was employed during 
the formulation of the reconstruction algorithm. The problem was approached using an iterative optimization method [8], [18]. The objective of the inverse EC problem here was to minimize the discrepancies between the model and the measured data with respect to the graphite conductivity values in the least squares sense. Therefore, the objective function of the problem was expressed in the generalized Tikhonov form but with an additional a priori knowledge based constraint (3).

$$
\begin{gathered}
\boldsymbol{\sigma}^{*}=\underset{\boldsymbol{\sigma}}{\operatorname{argmin}}\left\{g\left(\boldsymbol{\sigma}^{*}\right)=\frac{1}{2}\|\boldsymbol{r}(\boldsymbol{\sigma})\|^{2}+\frac{1}{2} \lambda\left\|\mathbf{L}\left(\boldsymbol{\sigma}-\boldsymbol{\sigma}_{\text {ref }}\right)\right\|^{2}\right\} \\
\text { for } \sigma_{L+1}>\sigma_{L}
\end{gathered}
$$

where $\sigma_{L}$ is the graphite model local conductivity corresponding to discretised layers, $\sigma_{L+1}$ is the local conductivity of the neighbouring layer as we move towards the outer brick periphery, $g\left(\boldsymbol{\sigma}^{*}\right)$ is the system objective function, $\boldsymbol{\sigma}^{*} \in \mathbb{R}^{n}$ is the discrete solution for the conductivity vector corresponding to $n$ graphite layers $\left(\boldsymbol{\sigma}^{*}=\right.$ $\left.\left(\sigma_{1}, \sigma_{2}, \sigma_{3} \ldots \sigma_{n}\right)^{T}\right), \boldsymbol{r}(\boldsymbol{\sigma})=\boldsymbol{F}(\boldsymbol{\sigma})-\boldsymbol{M} \in \mathbb{R}^{m}$ are the residuals between the forward model $\boldsymbol{F}(\boldsymbol{\sigma})$ and the data $\boldsymbol{M}$ measured at $m$ frequency values, $\boldsymbol{\sigma}_{\text {ref }}$ is a user-defined reference conductivity value, and $\lambda$ and $\mathbf{L}$ are the regularisation parameter and operator matrix respectively. It should be noted that the constraint in (3) essentially maintains a monotonic condition between the neighbouring graphite layers. The constraining approach could be enforced using various functions within the inversion algorithm depending on the available a priori knowledge. In fact, there are many alternative constraining techniques for general inverse problems, including upper and lower bounds, non-negativity or even taking a non-linear form such as quadratic constraints [23]. In this paper, the algorithm automatically imposes the monotonic constrain, that is the algorithm first checks if the imposed condition is satisfied, if not, it updates the solution based on the estimated conductivity values. During the reconstruction process the algorithm first compares the estimated neighbouring conductivity values $\sigma_{L+1}$ and $\sigma_{L}$ to see monotonic conditions are maintained between these local conductivity values. If $\sigma_{L+1}>\sigma_{L}$ a monotonic condition between neighbouring layers are maintained therefore the local solutions are accepted. However, if $\sigma_{L+1}<\sigma_{L}$ the monotonic condition is not maintained and hence algorithm will reject the estimated value for $\sigma_{L+1}$ and replace it with $\sigma_{L+1}=\sigma_{L+1}+\left(\sigma_{L}-\sigma_{L+1}\right)$ to satisfy the monotonic condition. It should be noted that the updated value for $\sigma_{L+1}$ is entirely based of the differences between the neighbouring local conductivities. This process will then continue until global monotonicity is achieved and used as an input for the forward and inverse solvers to check if it satisfied the gain ratio in (8) and the process will continue.

The general conductivity reconstruction problem aims to recover the unknown electrical conductivities of the material of interest from the boundary measurements, since the field distribution and the material conductivity are at the same time unknown, this makes the inverse EC problem highly nonlinear [8]. Furthermore, the general inverse EC problem is regarded as an ill-posed problem, mainly because the ECs decay rapidly with depth into the conducting material, which result in the sensitivity at the measurement boundary to be much larger than away from it. In the graphite reconstruction

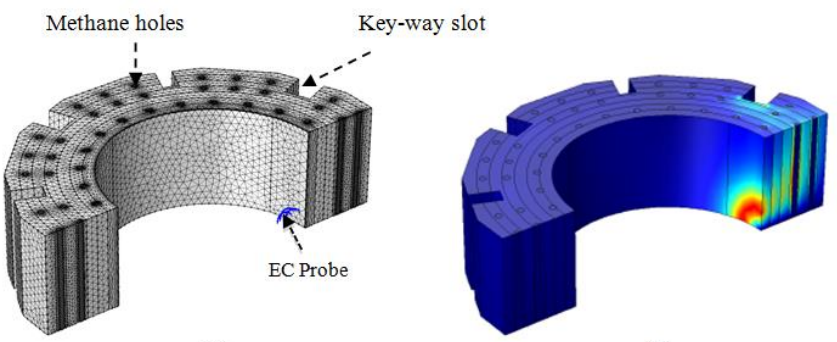

(a)

(b)

Fig. 2. One-quarter symmetry of the cylindrical AGR fuel channel graphite brick forward model geometry consisting of axial methane-holes, key-way slots and one of the gradiometer sensor: (a) FE mesh distribution and (b) EC distribution.

problem context, this means that the sensitivity at the bore will be much larger than the periphery, resulting in the sensitivity matrix (J) close to becoming singular [8]. Therefore, inverting J could lead to unstable solutions. To deal with these problems, an iterative RLM algorithm was employed (4). The RLM algorithm has been used previously by other authors to reconstruct shapes of low-conductivity objects within Magnetic Induction Tomography (MIT) [24]. In this paper, the RLM algorithm has been further formulated to make it suitable for the higher conductivities encountered in this graphite inverse problem and also to take in to account of a priori knowledge about the laboratory sample or the AGR fuel channel bricks internal electrical conductivity distributions.

$$
\begin{aligned}
& \delta \boldsymbol{\sigma}=-\left(\mathbf{J}_{k}^{T} \mathbf{J}_{k}+\gamma_{k} \mathbf{I}+\lambda \mathbf{L}^{T} \mathbf{L}\right)^{-\mathbf{1}} \mathbf{J}_{k}^{T}\left(\mathbf{F}\left(\boldsymbol{\sigma}_{\mathrm{k}}\right)-\mathbf{M}\right)+ \\
& +\lambda \mathbf{L}^{T} \mathbf{L}\left(\boldsymbol{\sigma}_{\mathrm{k}}-\boldsymbol{\sigma}_{\text {ref }}\right) \\
& \boldsymbol{\sigma}_{\mathrm{k}+1}=\boldsymbol{\sigma}_{k}+\delta \boldsymbol{\sigma}
\end{aligned}
$$

where $\delta \boldsymbol{\sigma}$ is the calculated change in conductivity, $\boldsymbol{\sigma}_{\mathrm{k}+1}$ is the updated conductivity value, $\gamma$ is a damping parameter, which is updated during the iterations, the subscript $k$ indicates the number of the iteration and $\boldsymbol{\sigma}_{k}$ and $\mathbf{J}_{k}$ are the conductivity and the Jacobian respectively computed in the $k^{t h}$ iteration.

The updating procedure of the damping parameter $\gamma$ is controlled through the calculation of the gain ratio $\rho$. In this context, the gain ratio refers to the ratio between the reduction in the Tikhonov and model objective functions [24]-[25]. To illustrate this let us define the Taylor series expansion of the objective function (6) and a quadratic model $M(\delta \boldsymbol{\sigma})$ to represent the behavior of the objective function $g(\sigma)$ in the neighbourhood of the current iteration (7).

$$
\begin{aligned}
& g(\boldsymbol{\sigma}+\delta \boldsymbol{\sigma})=g(\boldsymbol{\sigma})+\delta \boldsymbol{\sigma}^{T} \nabla g(\boldsymbol{\sigma})+\frac{1}{2} \delta \boldsymbol{\sigma}^{T} \nabla^{2} g(\boldsymbol{\sigma}) \delta \boldsymbol{\sigma}+ \\
& +\mathcal{O}\left(\|\delta \boldsymbol{\sigma}\|^{3}\right) \\
& M(\delta \boldsymbol{\sigma})=g(\boldsymbol{\sigma})+\delta \boldsymbol{\sigma}^{T} \nabla g(\boldsymbol{\sigma})+\frac{1}{2} \delta \boldsymbol{\sigma}^{T} \nabla^{2} g(\boldsymbol{\sigma}) \delta \boldsymbol{\sigma}
\end{aligned}
$$

As can be seen in equations (6) and (7), the quadratic model $M(\delta \boldsymbol{\sigma})$ is only a good approximation to the Taylor series 
expansion of the original objective function $g(\boldsymbol{\sigma}+\delta \boldsymbol{\sigma})$ up to the second order term, or in order for the model to have good approximation of the original objective function the term $\delta \boldsymbol{\sigma}$ must be small. Therefore, the gain ratio that governs the update of the damping parameter $\gamma$ is implemented using (8).

$\rho=\frac{g\left(\sigma_{\mathrm{k}}\right)-g\left(\sigma_{\mathrm{k}}+\delta \sigma\right)}{M(0)-M(\boldsymbol{\delta} \sigma)}$

where $\rho$ is the gain ratio, $g\left(\boldsymbol{\sigma}_{\mathrm{k}}\right)-g\left(\boldsymbol{\sigma}_{\mathrm{k}}+\delta \boldsymbol{\sigma}\right)$ is the reduction in Tikhonov objective function in (3) and $M(0)-$ $M(\delta \boldsymbol{\sigma})=-\delta \boldsymbol{\sigma}^{T} \nabla g(\boldsymbol{\sigma})-\frac{1}{2} \delta \boldsymbol{\sigma}^{T} \nabla^{2} g(\boldsymbol{\sigma}) \delta \boldsymbol{\sigma}$ is the difference between the model objective functions. A large value of $\rho$ corresponds to small value of $\delta \boldsymbol{\sigma}$, and hence the model $M(\delta \boldsymbol{\sigma})$ is said to be a good approximation to $g\left(\boldsymbol{\sigma}_{\mathrm{k}}+\delta \boldsymbol{\sigma}\right)$. Therefore the damping parameter $\gamma$ can be reduced. When $\rho$ is small it means that the model $M(\delta \boldsymbol{\sigma})$ is poor approximation to $g\left(\boldsymbol{\sigma}_{\mathrm{k}}+\delta \boldsymbol{\sigma}\right)$, and hence the damping parameter $\gamma$ can be increased. Negative values of $\rho$ implies that the numerator is negative suggesting that the objective function is not pointing in the descent direction; therefore the calculated solution in this case can be rejected. For a detailed description of equation (8) the reader is referred to [24]-[25].

The iterative RLM algorithm is essentially the extended version of the Regularized Gauss Newton (RGN) method presented in the previous publications for graphite inverse problem [16]-[18] and has a unique property that allows it to switch between the RGN and Gradient Descent (GD) method, through the update of a damping parameter.

Given the conductivity update in (4) and (5), for a very large value of damping parameter $\gamma \gg \operatorname{diag}\left(\mathbf{J}^{T} \mathbf{J}+\lambda \mathbf{L}^{T} \mathbf{L}\right)$ the updated solution corresponds to:

$\boldsymbol{\sigma}_{\mathrm{k}+1}=\delta \boldsymbol{\sigma}-\frac{1}{\gamma}\left(\left(\mathbf{F}\left(\boldsymbol{\sigma}_{\mathrm{k}}\right)-\mathbf{M}\right)+\lambda \mathbf{L}^{T} \mathbf{L}\left(\boldsymbol{\sigma}_{\mathrm{k}}-\boldsymbol{\sigma}_{\text {ref }}\right)\right)$

Whereas for small value of damping parameter $\gamma$ the conductivity update equation could be rewritten as:

$\boldsymbol{\sigma}_{\mathrm{k}+1}=\delta \boldsymbol{\sigma}-\left(\mathbf{J}^{T}{ }_{k} \mathbf{J}_{k}+\lambda \mathbf{L}^{T} \mathbf{L}\right)^{-1} \mathbf{J}^{T}{ }_{k}\left(\mathbf{F}\left(\boldsymbol{\sigma}_{\mathrm{k}}\right)-\mathbf{M}\right)+\lambda \mathbf{L}^{T} \mathbf{L}\left(\boldsymbol{\sigma}_{\mathrm{k}}-\right.$ $\left.-\sigma_{r e f}\right)$

It should be noted that (9) is equivalent to the GD equation, but in this case with side constraints term $\left(\lambda \mathbf{L}^{T} \mathbf{L}\left(\boldsymbol{\sigma}_{\mathrm{k}}-\boldsymbol{\sigma}_{\text {ref }}\right)\right)$, whilst (10) is equivalent to RGN method [16]-[18].

\section{Methodology}

As briefly mentioned in Section II, the forward model must be implemented such that it best represents the physical system. In some cases, it is also necessary to calibrate the model with measurements collected from a sample with known electrical properties. The necessity of the model calibration mainly arises to take into account the measurement errors caused by different experimental setups in laboratory and reactor conditions. In reality it is difficult to achieve 100 $\%$ model calibration or to have a zero error between the calibration and simulated model data.
For this reason, the preliminary study was mainly focused around the reconstruction of the graphite profiles using simulated datasets consisting of different artificially generated error levels. This approach serves to examine the effects of different error levels on accuracy of the reconstructed solutions, and therefore to determine the required levels of model calibration whilst assessing the robustness of the reconstruction algorithm. To generate the required datasets, first the error-free synthetic MF data was extracted from the graphite model after running the model using the known conductivity distribution of the laboratory sample. Two different types of errors were generated, namely random errors and replicated errors (errors generated based on the observed difference between measured data from laboratory sample and finite element prediction). Both errors were then distributed over the error-free synthetic data after scaling the errors with different percentage of the error-free data. Fig. 3a and Fig. 3b illustrate the different percentages of errors considered in this study.

During the inversion process of the synthetic datasets, constant regularization parameter and difference operator matrices $\left(L_{i j}=-1\right.$ for adjacent $i$ and $j, \quad L_{i j}=$ $\operatorname{diag}\left(u_{i}\right)$ for $i=j$ and $L_{i j}=0$ otherwise, where $u_{i}$ is the order of the operator) were used. The main reason for this choice was to evaluate the effects of data errors on the reconstructed profiles by maintaining all other parameters constant. After inverting the synthetic datasets, the reconstruction process was extended to invert data measured from a laboratory sample with known electrical conductivity distribution. This process was then followed by inverting multiple datasets collected from one of the operating AGR reactor.

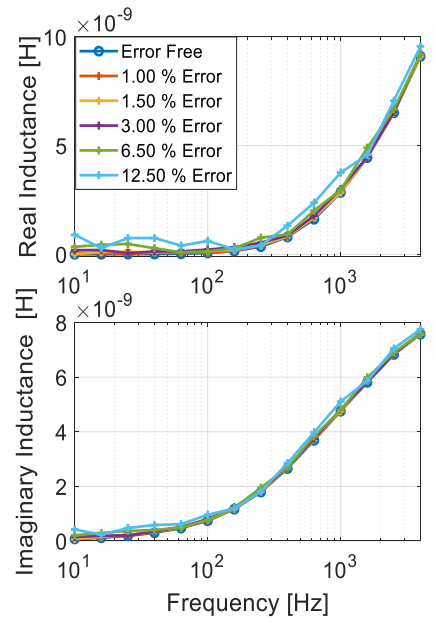

(a)
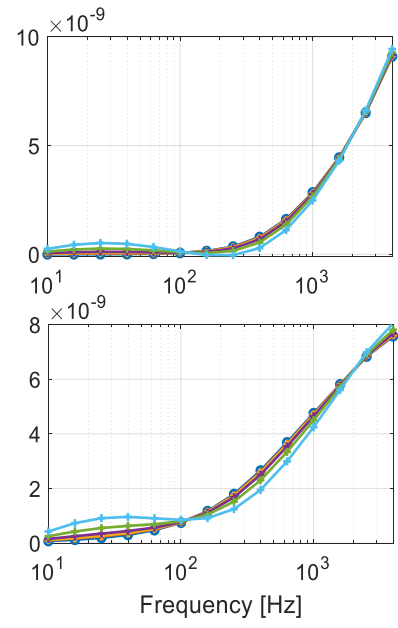

(b)
Fig. 3. An illustration of the investigated data errors that are distributed on the error-free data, (a) random errors added on the synthetic data, (b) replicated data error added on the synthetic data. It should be noted that the replicated data errors are generated based on the observed difference between measured data from laboratory sample and finite element prediction. 
We used Solartron 1260 impedance analyzer to measure the graphite brick response in both laboratory and reactor condition. During the measurements gradiometer sensor was aligned against the graphite brick bore and injected the exciter coil with signal consisting of frequency ranging between 10 $\mathrm{Hz}$ and $10 \mathrm{kHz}$ with 5 point per decade in the log-scale, and measured the responses due to the graphite being inspected. We set the lower frequency band to $10 \mathrm{~Hz}$ to increase the probe sensitivity to variations in electrical conductivity at the at the graphite brick outer periphery, whereas the upper frequency band was chosen to limit the effect of parasitic capacitance. The impedance analyzer has $0.1 \%$ measurement

TABLE I

THE PHYSICAL COIL PARAMETERS USED TO COLLECT THE MULTIFREQUENCY EDDY CURRENT DATA

\begin{tabular}{cccc}
\hline & $\begin{array}{c}\text { Pick-up } \\
\text { (Rx1) }\end{array}$ & Exciter (Tx) & $\begin{array}{c}\text { Backing-off } \\
(\mathrm{Rx} 2)\end{array}$ \\
Inner Diameter & $50 \mathrm{~mm}$ & $50 \mathrm{~mm}$ & $50 \mathrm{~mm}$ \\
Outer Diameter & $70 \mathrm{~mm}$ & $70 \mathrm{~mm}$ & $70 \mathrm{~mm}$ \\
Lift-off & $6 \mathrm{~mm}$ & - & - \\
Separation & - & $3 \mathrm{~mm}$ & $9 \mathrm{~mm}$ \\
Number of turns & 356 & 178 & 356 \\
\hline
\end{tabular}

accuracy, but post measurement analysis shows smoothly varying errors on the measured data, which may be caused by thermal drift components of the measurement system. Table I and Fig. 5 shows the coil parameters and geometry used for MF measurement, whereas Fig. 4 shows the simplified reconstruction algorithm block diagram employed to reconstruct the graphite brick radial conductivity profiles as a function of depth from the bore surface.

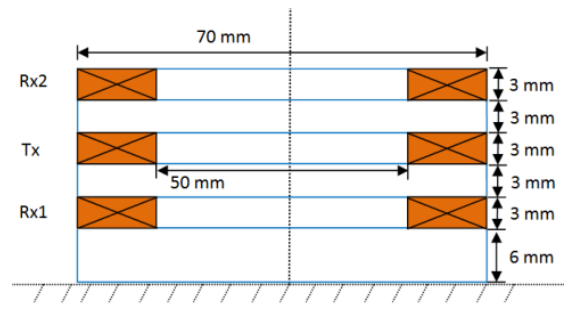

Fig. 5 Coil geometry used to collect multi-frequency eddy current data

It should be noted that the one-step inverse solver in Fig.4 refers to a non-iterative single-step inversion procedure to get a coarse solution often known as initial estimate. The main advantage of the one-step solver is that it is fast and requires only the solution of two forward models. However, for a more complex non-linear problem such as the one presented in this paper the one-step inverse solver could not be used, instead a full non-linear solver as shown in the entire block diagram need to be used.

\section{RESULTS AND DISCUSSION}

Fig. 6a and Fig. 6b show the reconstructed profiles of the simulated graphite brick using six datasets (shown in Fig. 3a and Fig.3b), each contaminated with different error levels ranging between $1 \%$ and $3 \%$ for both random and replicated error distributions. As would be expected, the most representative reconstruction of the true profile was obtained when the smallest amount error was applied to both datasets (1 $\%$ error). A similar trend can also be seen in Fig. $6 \mathrm{c}$ and Fig. $6 \mathrm{~d}$, where the objective function settles at the lowest value for

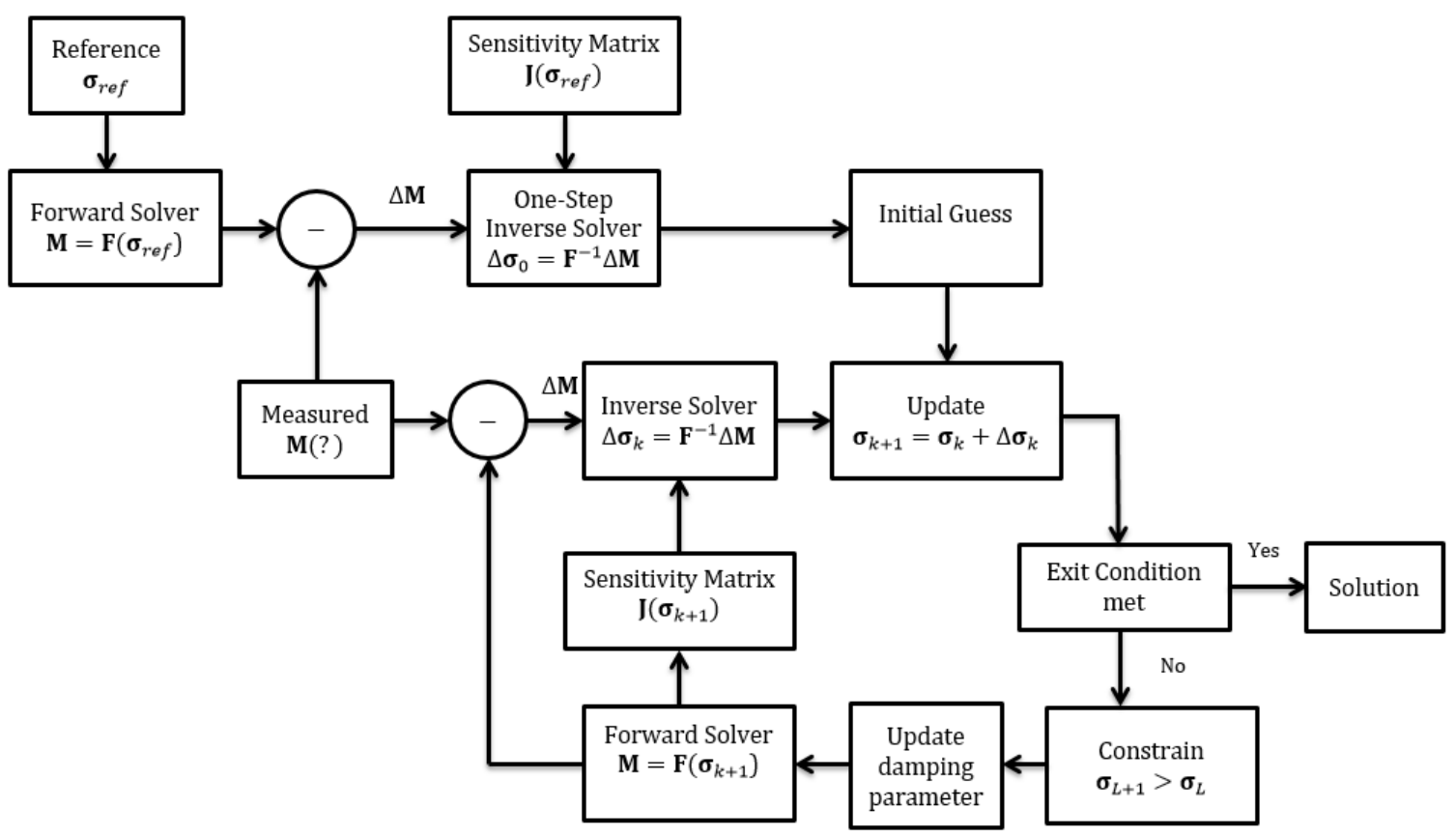

Fig. 4. The reconstruction algorithm block diagram that illustrates the inversion method employed to reconstruct the AGR fuel channel bricks radial conductivity profiles as a function of distance from the brick bore. 
the solution corresponding to the data with the least errors, and increases according to the amount of error applied to the original synthetic data. This is what should be expected since the objective function evaluates the norm of the residual errors, which were deliberately set to increase in this case. As the error level applied to the original data was increased, the

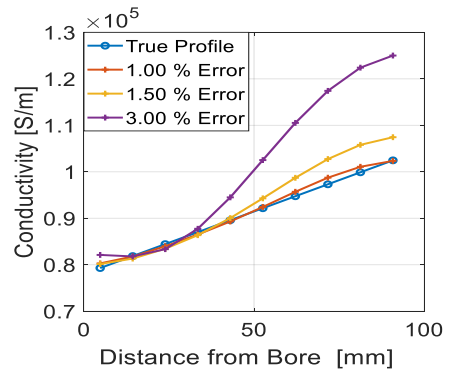

(a)

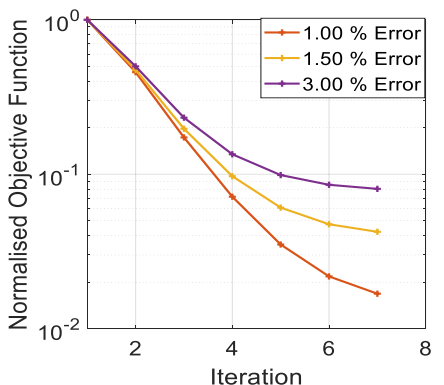

(b)

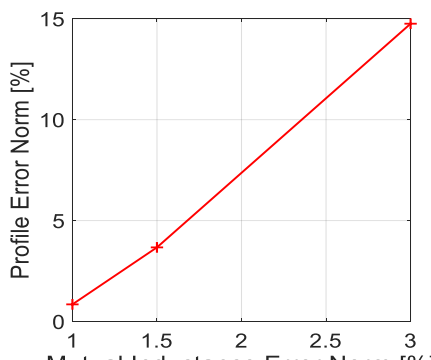

Mutual Inductance Error Norm [\%]

(e)

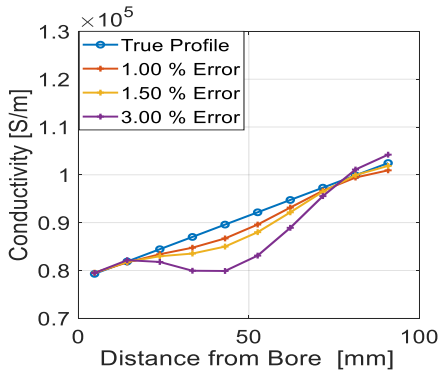

(b)

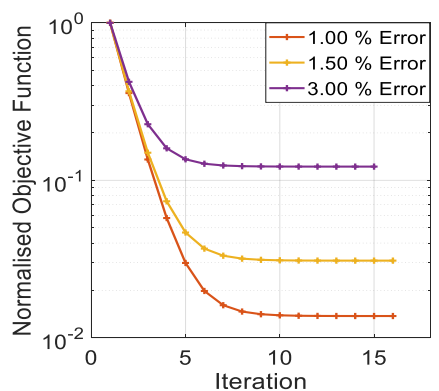

(d)

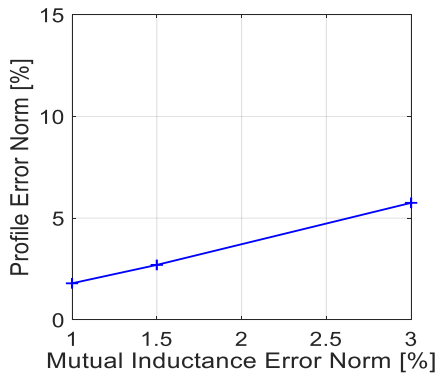

(f)
Fig. 6. The reconstructed profiles using simulated data with different levels of random data errors and replicated errors. (a) reconstructed profiles using random errors, (b) reconstructed profiles using replicated errors (c) objective function reduction for (a), (d) objective function reduction for (b), (e)data error verses profile error for (a) and (f) data error verses profile error for (b)

reconstructed profile diverged away from the exact solution, suggesting that the data were no longer representative of the original data that corresponded to the true profile. However, the divergence of the solution away from the exact solution with increasing error level differs for the two error distributions. This clearly suggests that the accuracy of the solution not only depend upon the error level, it is also significantly affected by the type of error distribution in the data.

The global profile errors reconstructed using the two error distributions were also found to increase in a linear fashion against the global errors of the input data (Fig. 6e and Fig. 6f). The smallest random error considered in this paper (1\% error) produced $\approx 1 \%$ global profile error, whereas the replicated data with identical error level produced $\approx 2 \%$. As the error levels increased to both datasets the profile errors caused by random data errors increased rapidly compared with the errors caused by the replicated datasets. The rapid increase in the profile errors when using the random error distribution compared with replicated data errors could be due to the distributions of the random errors, which have inconsistent variation over the frequency spectra, causing the algorithm to poorly fit the data during the reconstruction process.

The reconstructed near-bore conductivity solution using the random and replicated datasets show profile errors within a $1 \%$ range except for a data with random errors of $3 \%$ which shows $3.4 \%$ bore conductivity error, suggesting that the errors in the data were not significantly affecting the accuracy of the reconstructed bore conductivity values. The level of accuracy seen around the brick bore region is what should be expected in reality, as the sensitivity to a change in conductivity is much greater next to the probe than at the outer periphery.

Generally speaking, data errors more than $1.5 \%$ affected

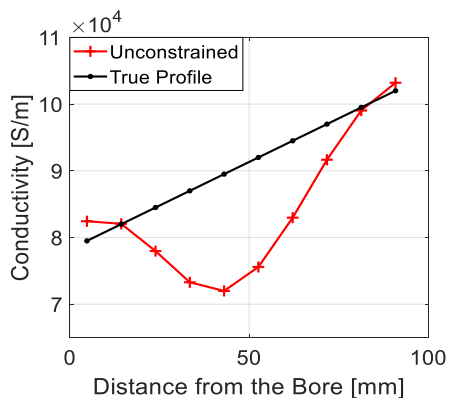

(a)

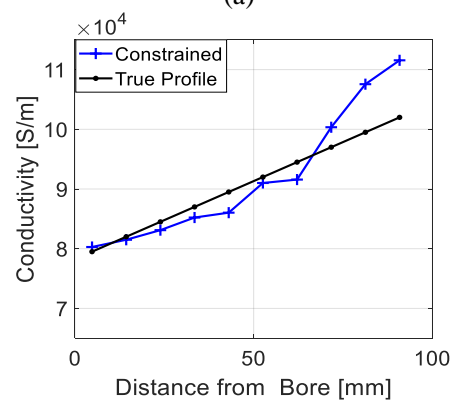

(c)

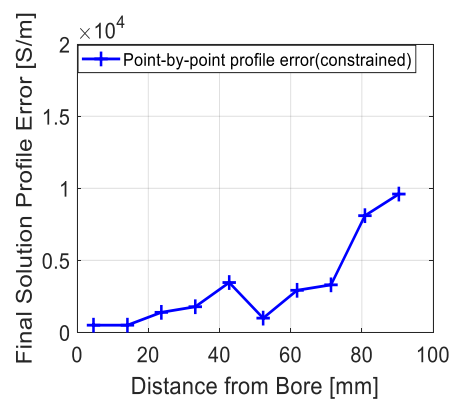

(e)

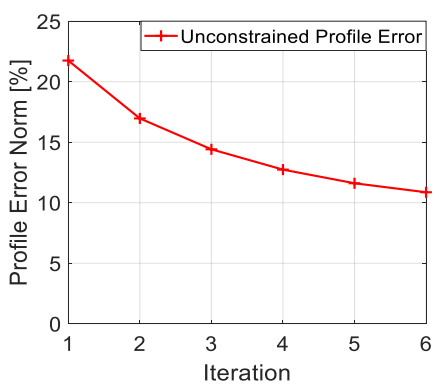

(b)

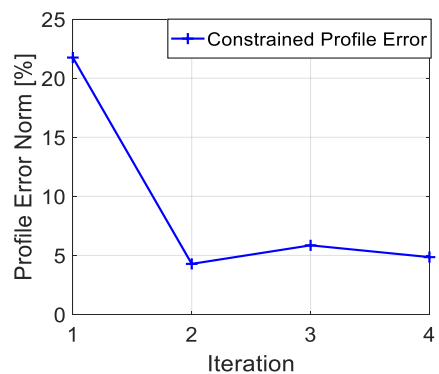

(d)

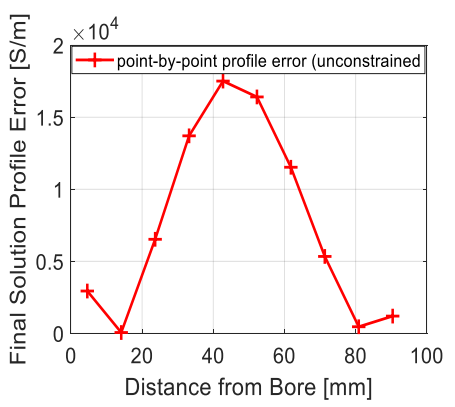

(f)
Fig. 7. Reconstructed conductivity profiles of a sample graphite brick using RLM algorithm along with constrained and unconstrained approaches. (a) unconstrained profile, (b) profile errors of (a), (c) constrained profile, (d) profile errors of (c), (e) the final solution profile error for (a) and (f) the final solution profile error for (c). it should be noted the plots in figure $7 \mathrm{e}$ and $7 \mathrm{f}$ shows the point-by-point profile errors of the final solutions whereas figure $7 \mathrm{~b}$ and $7 \mathrm{~d}$ shows the mean squared profile errors as a percentage at every iteration. 
TABLE II

UNCONSTRAINED AND CONSTRAINED RECONSTRUCTED PROFILES USING THE MEASUREMENTS TAKEN FROM LABORATORY SAMPLE

\begin{tabular}{ccc}
\hline Inverted Profiles & $\begin{array}{c}\text { Global Conductivity } \\
\text { Profile error }(\%)\end{array}$ & $\begin{array}{c}\text { Bore Conductivity } \\
\text { error }(\%)\end{array}$ \\
Unconstrained & 10.80 & 3.70 \\
Constrained & 3.36 & 1.00 \\
\hline
\end{tabular}

the reconstructed solutions away from the bore (Fig. 6a and Fig.6b), however the level of the profile error is also dependent upon the error distributions in the data. Therefore, in order to allow accurate reconstruction of the true profile, particularly in the presence unknown data errors additional a priori information about the sample of interest may need to be incorporated within the algorithm to constrain the solution based on the known information. An a priori knowledge based

approach may also be useful when model calibration with measurement collected from a sample with known conductivity distribution contains a large calibration error, or in this case a calibration error more than $1.5 \%$ of the original data.

The results in Fig. 7 and Table II show two of the profiles reconstructed using experimentally measured data from a brick with known electrical conductivity distributions, and the corresponding profile errors. In this case a brick was manufactured containing a matrix of radially drilled holes $\varnothing 4$ $\mathrm{mm}$ on a $180^{\circ}$ azimuth and $250 \mathrm{~mm}$ axial grid. This produced a grid with an apparent electrical conductivity, which increased with radius.

The two profiles in Fig. 7a and Fig. 7c were reconstructed using identical datasets, but reconstructed with and without the monotonic constraining technique discussed in Section II. It should be noted that the monotonic condition was chosen based on a priori knowledge we had about the drilled graphite brick, which is an increasing conductivity values from the graphite bore to periphery. This condition is also valid for some (but not all) of the AGR bricks due to the higher rate of diffusion of the $\mathrm{CO}_{2}$ coolant and the amount of radiation dose in the graphite bore regions, compared to the brick periphery..

The experimentally measured data unconstrained solution in Fig. 7a shows more or less identical trends as those reconstructed with replicated errors in Fig. 6b. The unconstrained bore conductivity value estimated the true profile with reasonable accuracy (3.70 \% error), although the profile generally shows a reducing trend with distance from the graphite bore before moving towards the true profile around the graphite periphery. The global profile error achieved for the unconstrained solution was $10.80 \%$. On the other hand, the constrained profile reproduced the true profile with much higher accuracy, ranging between $1.00 \%$ and 3.36 $\%$ for bore and global errors respectively (Table II). A possible explanation for the differences seen between the two solutions may be that as in the profiles reconstructed using simulated datasets (Fig. 6b); the unconstrained solution in Fig. 7a may have converged to a false local minimum or progressed to wrong solution. On the other hand, the

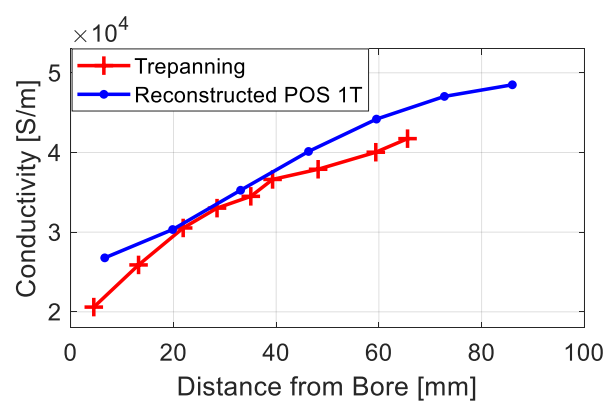

(a)

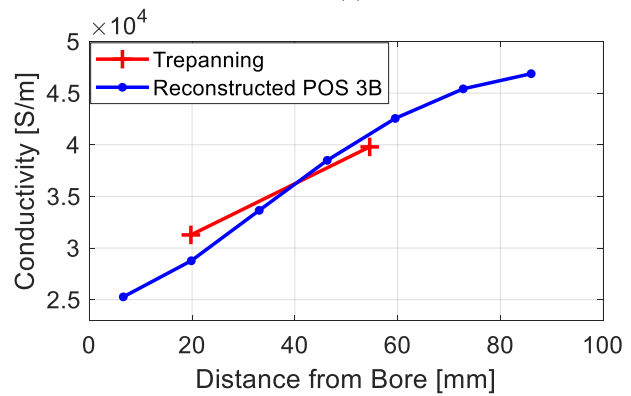

(b)

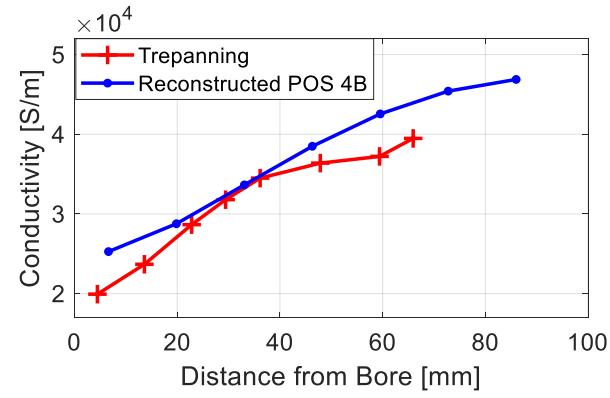

(c)

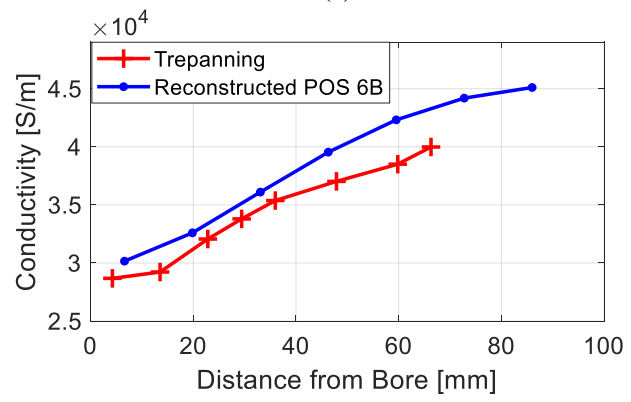

(d)

Fig. 8. Reconstructed conductivity profiles of the AGR graphite bricks using the measurements collected from one of the operating reactors. Note: the $\mathrm{x}-$ axis of the plots show the distance from the graphite bore to the middle of each graphite domain in the case of the reconstructed profiles, whilst the trepanning show the conductivity values deduced from the density measurements after slicing the trepanned samples.

TABLE III

COMPARISON BETWEEN THE MEAN VALUES OF TREPANNED SAMPLE AND THE RECONSTRUCTED PROFILES

\begin{tabular}{cccc}
\hline $\begin{array}{c}\text { Inspected } \\
\text { Locations }\end{array}$ & $\begin{array}{c}\text { Mean Profile } \\
\text { Value } \\
(\mathrm{S} / \mathrm{m})\end{array}$ & $\begin{array}{c}\text { Mean Trepanning } \\
\text { Measurement }(\mathrm{S} / \mathrm{m})\end{array}$ & $\begin{array}{c}\text { Mean } \\
\text { Profile } \\
\text { Error (\%) }\end{array}$ \\
POS 1T & 37209.30 & 31924.44 & 14.20 \\
POS 3B & 35555.56 & 35020.13 & 1.50 \\
POS 4B & 35978.99 & 29912.36 & 16.90 \\
POS 6B & 39544.44 & 33879.93 & 14.30 \\
\hline
\end{tabular}


constrained solution may have avoided this through the monotonic constraint applied to it.

Fig. 8 and Table III show the reconstructed conductivity profiles of the AGR fuel channel bricks using the measurements collected from one of the operating AGR reactor. For a validation purpose the reconstructed profiles were compared against the conductivity measurements of the $\varnothing 19 \mathrm{~mm}$ by $75 \mathrm{~mm}$ trepanned cylindrical samples machined out of the same fuel channel locations where the eddy current data were acquired.

Two main features can be seen in these plots. The first is that the monotonic nature of the trepanned conductivity measurement as a function of depth from the graphite bore validates (at least in this case) the suitability of the monotonic constrain applied within the algorithm during the reconstruction process.

The second and more important feature is that the algorithm has produced practical profiles with the correct order of magnitude, which agreed reasonably well with the measured conductivity values of the trepanned samples throughout their entire depth profile. Nevertheless, looking into the details of each plot in Fig. 8 reveals that the bore conductivity values of the trepanned samples in Fig. 8a and 8c are smaller than those reconstructed from the EC data. These differences in bore conductivity values between the measured and reconstructed profiles may be due to different factors. One of the factors could be the amount of graphite brick volumetric information captured from the trepanned samples. In other words, the trepanned samples covered only a small $\varnothing 19 \mathrm{~mm}$ by $75 \mathrm{~mm}$ cylindrical section of the graphite bricks, whereas the EC measurements interrogated a larger proportion of the graphite volumetric information because of the spatial extent of the EC flow inside the brick. Since the radiolytic oxidation of the AGR bricks has a localized effect, the measured bore density/conductivity from the cylindrical trepanned samples might not be exactly identical to the overall bore density/conductivity values. However, there is currently no other means of validating the reconstructed conductivity profiles, and hence the trepanning sample measurements are the only means of validation for the conductivity profiles.

Table III shows the mean profile errors between the measured and reconstructed conductivity values. Despite the differences in some of the bore conductivity values, the algorithm has produced reasonable estimates of the trepanned sample conductivity values for the fuel channel bricks studied in this paper. The reconstructed profile errors range between $1.5 \%$ and $16.90 \%$ suggesting that the algorithm has operated as it should. The only drawback of the RLM algorithm presented in this paper is that it takes several hours to arrive at the finale solution when carried out on a workstation with 48 GB RAM. This was mainly due to the computations involved in the trials to ensure that the objective function is always reducing through the continual updating of the damping parameter.

\section{CONCLUSION}

Monitoring the condition of the graphite cores in AGR's is one of the safety case requirements for continued operation of the reactors. There are several in-core graphite properties that are being monitored in order to implement robust safety cases. One of these graphite properties is the graphite volumetric density/conductivity variation under irradiation and radiolytic oxidation. This paper has presented a graphite depth profiling approach using a nonlinear inverse EC method. The modified RLM algorithm for reconstructing the complex structured fuel channel brick electrical conductivity profiles was presented and tested using a laboratory sample and the datasets collected from one of the operating reactors. This method uses a damping parameter, which was updated during the iteration, and hence could work fairly well even with less optimal regularization parameter. The reconstructed fuel channel brick profiles were compared against conductivity measurements from trepanned samples taken out of the reactor core at the same locations where the data for reconstruction were collected. It was found that the algorithm has produced reasonable estimates of the trepanned sample conductivity trends, with the mean profiler errors ranging between $1.5 \%$ and $16.9 \%$. This is the first time that the AGR fuel cannel brick conductivity profiles are reconstructed with such accuracy, and currently more work is being carried out to further improve the speed and accuracy of the reconstruction procedure. The study has also demonstrated that error levels and distributions affect the accuracy of the reconstructed profiles in different ways, and in order to accurately estimate the graphite volumetric conductivity profiles, the measurement errors in the data need to be sufficiently small or an additional constraining mechanism may need to be implemented within the reconstruction algorithm.

\section{ACKNOWLEDGMENTS}

The authors would like to thank the UK Engineering and Physical Science Research Council and EDF Energy for funding this project under EPSRC IAA 122.

\section{REFERENCES}

[1] Steer, A.G. "AGR Core Design, Operation and Safety Functions." Management of Ageing in Graphite Reactor Cores. Cambridge: Royal Society of Chemistry. ISBN: 0854043454, (2007):11-18.

[2] Kelly, B. T., Marsden, B. J., Hall, K., Martin, D. G., Harper, A., and Blanchard, A. "Irradiation damage in graphite due to fast neutrons in fission and fusion systems." IAEA Tecdoc, (2000),1154.

[3] Simmons, J. H. W. "Radiation Damage in Graphite." International Series of Monographs in Nuclear Energy. Vol. 102. Elsevier, (2013).

[4] Hacker, P.J., Neighbour G.B., and McEnaney B. "The coefficient of thermal expansion of nuclear graphite with increasing thermal oxidation.” Journal of Physics D: Applied Physics 33.8 (2000): 991.

[5] Bloodworth T., Anderson M., Brown M. and Williams J. "Eddy current inspection of AGR fuel channels: keyway cracks and density mapping." In: Proc. NDT, Northamptonshire, UK, (2012).

[6] García-Martín, J., Gómez-Gil, J., and Vázquez-Sánchez, E. "Nondestructive techniques based on eddy current testing." Sensors, (2011), 11(3), 2525-2565.

[7] Tesfalem, H., Fletcher, A. D., Brown, M., Chapman, B., \& Peyton, A. J. (2018). "Study of asymmetric gradiometer sensor configurations for eddy current based non-destructive testing in an industrial environment." NDT \& E International, 100, 1-10.

[8] Norton, S. J., Kahn, A. H. and Mester, M. L. "Reconstructing electrical conductivity profiles from variable-frequency eddy current measurements." Journal of Research in Nondestructive Evaluation, 1(3), (1989):167-179

[9] Yin, W, Dickinson, S.J. and Peyton, A. J. "Imaging the continuous conductivity profile within layered metal structures using inductance spectroscopy." IEEE Sensors Journal (2005). 5.2: 161-166. 
[10] Bowler J. R. and Stephen J. N. "Eddy current inversion for layered conductors." Journal of Research in Non-destructive Evaluation 4.4 (1992), pp 205-219.

[11] Yin, W., Dickinson, S. J., \& Peyton, A. J. (2006). Evaluating the permeability distribution of a layered conductor by inductance spectroscopy. IEEE transactions on magnetics, 42(11), 3645-3651.

[12] Yin, W., \& Peyton, A. J. (2006, April). Determining the Step-change Conductivity Profile within Layered Metal Structures Using Inductance Spectroscopy. In 2006 IEEE Instrumentation and Measurement Technology Conference Proceedings (pp. 2127-2131). IEEE.

[13] Moulder, J. C., Uzal, E., \& Rose, J. H. (1992). Thickness and conductivity of metallic layers from eddy current measurements. Review of scientific instruments, 63(6), 3455-3465.

[14] Norton, S. J., \& Bowler, J. R. "Theory of eddy current inversion". Journal of Applied Physics, (1993), 73(2), 501-512.

[15] Uzal, E., Moulder, J. C., Mitra, S., \& Rose, J. H. (1993). Determining conductivity and thickness of continuously varying layers on metals using eddy currents. In Review of Progress in Quantitative Nondestructive Evaluation (pp. 251-258). Springer, Boston, MA.

[16] Dekdouk, B., Chapman, R., Brown, M., and Peyton, A. J. "Evaluating the conductivity distribution in isotropic polycrystalline graphite using spectroscopic eddy current technique for monitoring weight loss in advanced gas cooled reactors." NDT \& E International 51 (2012): 150159.

[17] Fletcher, A. D. "Non-destructive testing of the graphite core within an Advanced Gas-Cooled Reactor." Doctoral dissertation, The University of Manchester, Manchester, 2014.

[18] Tesfalem, H., Peyton, A., Fletcher, A. Brown, M. and Chapman, B. "Eddy Current Sensors and Inversion Techniques for Estimation of the Conductivity Profile of the Graphite Bricks in an Advanced Gas-cooled Reactor Core". IOS Press Book Volume 42: Electromagnetic Nondestructive Evaluation (XX). DOI: 10.3233/978-1-61499-767-2253, (2017), pp $253-264$.

[19] COMSOL Inc. "AC/DC Module: User's Guide". Version 5.2.

[20] Soleimani, M., Lionheart, W. R., Peyton, A. J., Ma, X., and Higson, S. R. "A three-dimensional inverse finite-element method applied to experimental eddy-current imaging data." IEEE Transactions on Magnetics 42.5 (2006): 1560-1567

[21] Yin, W, and Peyton, A.J. "Sensitivity formulation including velocity effects for electromagnetic induction systems". IEEE Transactions on Magnetics 46.5 (2010): 1172-1176.

[22] Dyck, D. N., Lowther, D. A. and Freeman, E. M. "A method of computing the sensitivity of electromagnetic quantities to changes in materials and sources". IEEE Transactions on Magnetics 30.5 (1994): 3415-3418.

[23] Nocedal J, and Wright, S. "Numerical optimization." Springer Science \& Business Media, (2006).

[24] Dekdouk, B., Ktistis, C., Armitage, D. W., and Peyton, A. J. “Absolute imaging of low conductivity material distributions using nonlinear reconstruction methods in magnetic induction tomography." Progress In Electromagnetics Research, 155, (2016), 1-18.

[25] Madsen, K., Nielsen, H. B., and Tingleff, O. "Methods for non-linear least squares problems." (1999).

[26] Bowler, N., \& Huang, Y. Electrical conductivity measurement of metal plates using broadband eddy-current and four-point methods. Measurement Science and Technology, (2005). 16(11), 2193.

[27] Carvalho, A. A., Rebello, J. M. A., Sagrilo, L. V. S., Camerini, C. S., \& Miranda, I. V. J. MFL signals and artificial neural networks applied to detection and classification of pipe weld defects. Ndt \& E International, (2006). 39(8), 661-667.

[28] Zhu, P., Cheng, Y., Banerjee, P., Tamburrino, A., \& Deng, Y. A novel machine learning model for eddy current testing with uncertainty. NDT \& E International, (2019). 101, 104-112.

HenokTesfalem received the M.Eng. degree in Electrical and Electronics engineering in 2014 and the Ph.D. degree in 2018 from the University of Manchester, Manchester, U.K. His Ph.D. thesis was titled "Eddy current based non-destructive testing of the graphite bricks in an advanced gas-cooled reactor core". He joined the University of Manchester as a post-doctoral research associate in April 2018. His research focuses in electromagnetic non- destructive testing including conductivity profiling and defect detection and characterization.

Anthony J. Peyton received the B.Sc. degree in electrical and electronics engineering in 1983 and the Ph.D. degree in 1986 from the University of Manchester, Manchester, U.K. His Ph.D. thesis was titled "Device for the assessment and rehabilitation of kinetic muscle function and circuitry for monitoring localized muscle fatigue." He was appointed as Principal Engineer at Kratos Analytical Ltd. in 1989, developing precision electronic instrumentation systems for magnetic sector and quadrupole mass spectrometers, from which an interest in electromagnetic instrumentation developed. He returned to University of Manchester as a Lecturer and worked with the Process Tomography Group. He moved to Lancaster University in 1996, taking up post of Senior Lecturer. He was promoted to reader in Electronic Instrumentation in July 2001 and Professor in May 2004. Since December 2004, he has been Professor of Electromagnetic Tomography Engineering at UMIST. His main research interests currently are in the area of instrumentation, applied sensor systems, and electromagnetics including nondestructive testing systems, ground penetrating radar systems, security scanning and landmine detection systems.

Adam D Fletcher received the MSci degree in Mathematics and Physics in 2008 from the University of Durham, UK and the EngD degree in Nuclear Engineering from the University of Manchester, UK in 2014. This PhD thesis was titled "Non-destructive Testing of the Graphite Core within an Advanced Gas-Cooled Reactor". He joined EDF Energy in 2014 as a Graphite Core Inspection Engineer implementing new techniques for AGR core inspection. He returned to Manchester University in 2019 as a Research Associate developing electromagnetic techniques for landmine detection. His main research interests are in electromagnetic non-destructive testing systems, sensor systems, inverse problems and computational electromagnetics.

\section{Matthew Brown}

Matthew Brown received a Ph.D. in Materials from UMIST, UK in 2002. His $\mathrm{Ph} . \mathrm{D}$. thesis was titled "Investigation of microwave plasma deposited silica coatings on carbon fibres used in aluminium matrix composites". He subsequently joined BNFL and worked at Berkeley Laboratory for four years on the characterisation of irradiated PGA graphite for Magnox reactors. He joined EDF-Energy in 2006 and has since worked on the development of graphite inspection techniques for AGR reactors 